\title{
COMPARACIÓN DE CLASIFICACIÓN DE MADERA ASERRADA DE PINO RADIATA POR EL MÉTODO SECUENCIAL, ESTRUCTURAL Y SHOP.
}

\section{JAVIER GONZÁLEZ MOLINA (*), ROSE MARIE GARAY MOENA (*), FERNANDO BUSTAMANTE FERRARI (**) y PABLO CROXATTO ALARCÓN $(* *)$.}

(*) Departamento de Tecnologia de la Madera. Universidad de Chile.

(**) Ingenieros Forestales. Mención Tecnología de la Madera

\section{RESUMEN}

El presente estudio tiene como objetivo clasificar la madera de las trozas aserrables de pino radiata, en tres categorias $A, B y C$, exterior, media y central, respectivamente. Sobre la base de esta clasificación se establecieron los productos que se pueden obtener de las trozas. Se utilizaron las normas de clasificación visual para uso estructural (NCH 1207. OF. 90), de piezas aptas para el uso en viviendas y la norma Factory Shop, que las clasifica en función de su aptitud, para la confección de partes y piezas de puertas y ventanas.

Como organización de la información se confeccionó el predictor FERPA, el que sobre la base de variables de entrada, conocidas facilmente por el usuario, como dominancia y clase diamétrica, se pueden establecer los volumenes $A, B y C$ de la troza en cuestión y establecer de esta manera, los productos a obtener por las normas ya expuestas.

Palabras clave: Clasificación madera, Pinus radiata.

\section{ABSTRACT}

The objective of this study is to define, inside the log of pino radiata, by secuencial norms in especifically $A, B$ and $C$ areas, exterior, midside and central part, respectively to establish the relationship between the products that it can be found. In this case, the pieces werw classified by visual procedures for estructural uses (NCH 1207. OF. 90), wich gives pieces of wood apropiate, to be used in houses's constructions and the Factory and Shop norm, what permits their classification, in order to make them useful in the construction of doors and windows.

In order to canalize the information, a Predictor FERPA, was created to operate with well known variables, dominancy and diametric class, wich permits estimate the volumes $A, B$ and $C$ of the logs and it's later classification, with the above mentioned procedures.

Keywnrds: Wood classification, Pinus radiata. 


\section{ANTECEDENTES GENERALES}

El sector madera aserrada y productos derivados de pino radiata, ha significado en el último quinquenio el segundo rubro de exportaciones forestales con un promedio anual, para el periodo, de más de US\$ 170 millones.

Una de las limitaciones para mayores exportaciones, en el sector, lo constituye el alto porcentaje de defectos en la madera, tales como nudos, pecas, médula, manchas y bolsas de resinas. El potencial de producción de madera de alta calidad, pasa por el conocimiento íntimo del recurso y las consecuencias que sobre él tienen, ciertas prácticas silvicolas como raleo y podas, principalmente.

Una forma de conocer el recurso madera es clasificar el bosque en pié y tratar de predecir la calidad de los distintos productos que de él se obtendrán. En este trabajo se expone el uso de un Predictor (FERPA). de caracterización tridimensional, el cual guarda una muy buena correlación con la norma estructural y la Schop.

\section{REVISIÓN BIBLIOGRÁFICA}

Pino radiata es una especic que presenta ramas muy pequeñas y verticilos cercanos, en los cuatro primeros metros de altura. Hasta los $15 \mathrm{~m}$ de altura, los nudos aumentan en tamaño y los verticilos se distancian progresivamente. Las ramas más largas se encuentran entre los 15 y $30 \mathrm{~m}$ de altura.

La zona del corazón del árbol se extiende hasta los $20 \mathrm{~m}$ y la zona de nudos muertos hasta $\operatorname{los} 22 \mathrm{~m}$ de altura.

La distribución de los conos unidos al tronco y ramas comienza a los $12 \mathrm{~m}$, generando perforaciones cilindricas de resinas, perpendiculares al eje central del árbol, desde la médula a la corteza, no asi las ramas que forman tejido, en la misma zona, con inclinaciones que dependen del ángulo de inserción de éstas (Lavery. 1986).

Tradicionalmente la madera aserrada se clasifica por su resistencia o por su apariencia. Si se atiende su resistencia, interesarán aquellas alteraciones de crecimiento que debiliten sus propiedades mecánicas, tamaño, ubicación y frecuencia. Esto exige caracterizar las cuatro caras de la pieza y sus extremos. Este criterio se utiliza en la madera para la construcción.

En una norma por aspecto, generalmente se clasifica la mejor cara y se caracterizan nudos, médula, bolsas de resinas o corteza. pecas y otros. Se usa esta clasificación, en la madera que se destina a revestimientos, molduras, marcos de puertas, ventanas y otros, (Wagner. 1992). En este caso sc trata de usos no estructurales. 
Las calidades Factory y Shop, se han desarrollado para dimensiones americanas, utilizadas en carpintería y madera aserrada. Se definen por el número de piezas limpias, que se pueden obtener, conforme a la norma, después de aserrar la pieza. Se utilizan normalmente en la industria secundaria, tales como fabricación de puertas, ventanas, mobiliario, marcos, molduras, cajas y ebanistería. El mejor producto es Shop 1, después 2 y 3 y finalmente Shop-out, que desclasifica (WWPA, 1990).

CORMA (Corporación Chilena de la Madera) define tres calidades estructurales, la calidad normal acepta piezas que son de gran capacidad resistente en la construcción. El control se realiza principalmente, sobre la base de tamaño, ubicación y frecuencia de nudos, específicamente la razón de área nudosa de borde (RANB), desarrollada inicialmente en Inglaterra (INN NCh 1207 of.90).

La clasificación secuencial, desarrollada en Forelco, por la Unidad de Estudios, distingue tres calidades de madera, según la ubicación de ésta, en el corte transversal de la troza aserrable.

- Calidad A: Madera de destape, presenta por lo general nudos muertos y un nudo por cara (a lo ancho de una tabla) y presenta sólo albura.

- Calidad B: Madera libre de médula, con alta proporción de nudos vivos, dos nudos a lo ancho de una cara y puede presentar duramen o albura.

- Calidad C: Presenta médula y mayor porcentaje de duramen (Frola, Bustamante y Croxatto, 1993).

Los nudos afectan la resistencia del material, ya que las fibras se desvian en su entorno, llegando a ser casi perpendiculares a la madera adyacente. El efecto sobre las propiedades mecánicas dependen de su tamaño, forma, adherencia y localización, siendo mayor o menor en función de la solicitud a la cual se someta (Kollmam, 1959). Cabe hacer notar que el efecto del nudo, cuyo diámetro varía entre 14 y $18 \mathrm{~mm}$, abarca una superficie de 2 a 4 veces su radio (Zandbergs, 1988).

Especialmente delicado es el caso de los nudos muertos o sueltos, procedentes del recubrimiento de muñones de ramas, posterior a la poda artificial o natural, pues se cubren con los nuevos anillos de crecimiento, quedando embutidos en éstos (Retamal, 1975).

Las manchas son producto de la policromias de hifas de hongos en la madera (Donoso, 1991). Varían en la coloración de negro-azulado a pardo-rojizo y anaranjado. Se pueden encontrar en albura de árboles recién cortados, en tablas en proceso de secado y, en general, en distintos productos forestales. La intensidad de los ataques aumenta con contenidos de humedad de la madera comprendidos entre 40 y $50 \%$. La 
madera con este tipo de ataque no disminuye su resistencia mecánica de manera apreciable (Boyce, 1961).

Estos hongos aumentan la permeabilidad de la madera, pueden reducir su peso especifico en 1 o $2 \%$, la resistencia a la compresión paralela al grano y el módulo de ruptura pueden disminuir hasta en $5 \%$ (Del Pozo, 1984). La mancha es perjudicial en la clasificación estética de la madera y causa gran desclasificación en productos aserrados (INFOR, 1989).

Las pecas son rastros de acículas en la madera. Se pueden clasificar como peca fuerte, de color oscuro y gran tamaño de elemento y peca débil, asociada a madera juvenil, de color claro y pequeñas (Frola, Bustamante y Croxatto, 1993).

Las bolsas de resinas son cavidades planas que aparecen por lo general dentro de un anillo de crecimiento. En pino radiata son frecuentes debido a sus canales resiníferos. Este defecto desclasifica la madera para ebanisteria. aún en duramen; la bolsa de resina conserva su color claro. alterando aún más su aspecto (Kollman,1959). Su presencia altera la resistencia a la tracción. compresión y cizalle (INFOR, 1979).

La médula se presenta en la parte central del tronco y está constituída por parenquima blando. Tiene poca importancia sobre las características mecánicas, pero es causa de desclasificación en madera elaborada. Puede ser de sección circular, poligonal o estrellada, su diámetro alcanza hasta los 16 mm (Kollman, 1959).

\section{MATERIAL Y MÉTODO}

Para efectos del muestreo se trabajó con un rodal de 26 a 28 años, con una distribución normal de arboles en el bosque: $45 \%$ dominante, $33 \%$ codominante y $22 \%$ intermedios (Forelco, 1992). Para los efectos prácticos se eliminaron del muestreo los suprimidos. ya que no aportan, sino marginalmente al volumen aserrable. Se usó un muestreo estratificado. con muestras proporcionales al tamaño del estrato.

La muestra consistió en $74 \mathrm{~m}^{3}$. de los cuales se usaron $14 \mathrm{~m}^{3}$, para el análisis tridimensional y los $60 \mathrm{~m}^{3}$ restantes, para la clasificación con las tres normas.

De la muestra aserrable de $60 \mathrm{~m}^{3}$, un $20 \%$ se consideró de internudo largo, es decir a $1,20 \mathrm{~m}$. Como caracterización de diferencia entre duramen y albura se consideró la presencia de resina en albura. De esta forma se determinó la diferencia de zonas A, B y C. Los nudos, para el modelaje tridimensional se consideraron de 7 formas: ovalado, redondo, lanceolado. ojo. alargado, cónico y en $\mathrm{V}$.

Las trozas se obtuvieron de $4.15 \mathrm{~m}$ de largo y se agruparon en 6 tipos, combinando grado de dominancia y largo de internudo $(3 \times 2)$. En cada grupo se distinguieron 4 clases diamétricas: $12-20 \mathrm{~cm}$. $22-30 \mathrm{~cm} .32-40 \mathrm{~cm}$ y $42-50 \mathrm{~cm}$. A continuación se pintó 
la albura, del diámetro menor de cada troza, para reconstituirla, posteriormente, a base de la clasificación secuencial.

En la práctica, los 24 grupos potenciales $(3 \times 2 \times 4)$ quedaron reducidos a 13 , con la siguiente caracterización:

Cuadro $\mathrm{N}^{\circ} 1$.

DISTRIBUCIÓN DE GRUPOS, DOMINANTES

\begin{tabular}{|c|c|c|}
\cline { 2 - 3 } \multicolumn{1}{c|}{} & \multicolumn{2}{c|}{ Internudos } \\
\hline Clase Diamétrica $(\mathrm{cm})$ & Largos, $\mathrm{N}^{\circ}$ del grupo & Cortos, $\mathrm{N}^{\circ}$ del grupo \\
\hline $12-20$ & - & 1 \\
\hline $22-30$ & 22 & 3 \\
\hline $32-40$ & 23 & 5 \\
\hline $42-50$ & 24 & 7 \\
\hline
\end{tabular}

Cuadro $\mathrm{N}^{2} 2$.

DISTRIBUCIÓN DE GRUPOS, CODOMINANTES

\begin{tabular}{|c|c|c|}
\cline { 2 - 3 } \multicolumn{1}{c|}{} & \multicolumn{2}{c|}{ Internudos } \\
\hline Clase Diamétrica $(\mathrm{cm})$ & Largos, $\mathrm{N}^{\circ}$ del grupo & Cortos, $\mathbf{N}^{\circ}$ del grupo \\
\hline $12-20$ & 4 & - \\
\hline $22-30$ & 6 & 11 \\
\hline $32-40$ & 8 & 13 \\
\hline
\end{tabular}

Cuadro No3.

DISTRIBUCIÓN DE GRUPOS, INTERMEDIOS

\begin{tabular}{|c|c|}
\cline { 2 - 2 } \multicolumn{1}{c|}{} & Internudos \\
\hline Clase Diamétrica $(\mathrm{cm})$ & Cortos, $\mathrm{N}^{\circ}$ del grupo \\
\hline 22.30 & 18 \\
\hline
\end{tabular}

La cubicación de las trozas se hizo a base de la norma JAS y el esquema de corte corresponde a cada clase diamétrica, extrayendo un cuartón central variable, entre $5 \times 2$ " y 6x6", sacando el máximo de tablas de 1 y 1,5" de espesor y en diferentes anchos.

Todas las tablas y cuartones se pintaron de un color en los extremos, en función del grupo. Una vez seca la madera, se cepilló por ambas caras y se clasificó en forma secuencial, Shop y estructural.

Otro de los objetivos, como se mencionó anteriormente, fue el poder predecir la cantidad de cada producto a obtener del bosque, sobre la base de un predictor "FERPA", teniendo en cuenta diámetro, grado de dominancia y tipo de internudo. Para ello se utilizaron los $14 \mathrm{~m}^{3}$ restantes (Figura $\mathrm{N}^{\circ} 1$ ). 

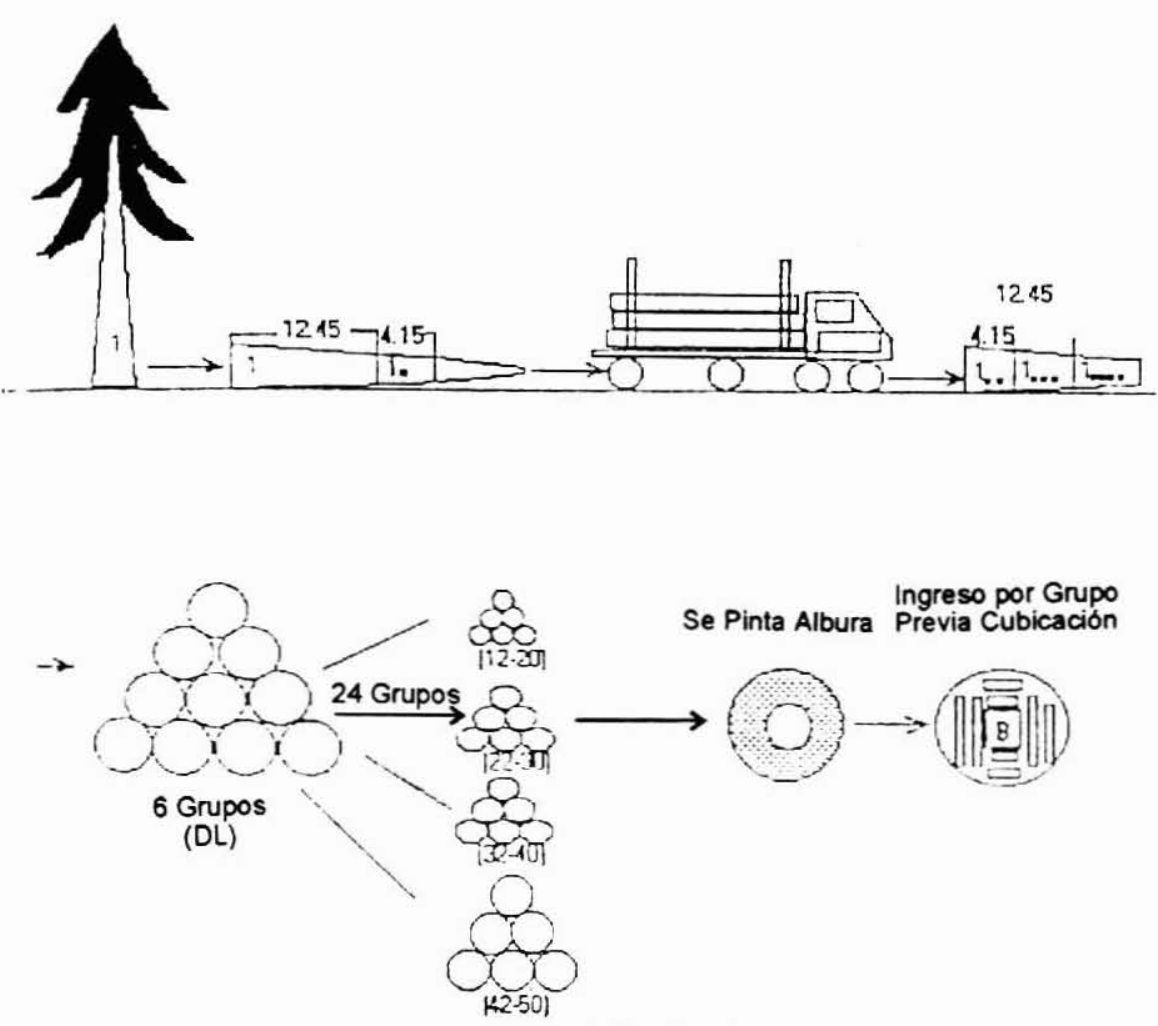

Cubicado y Clasificado

Bajo las 3 Normas

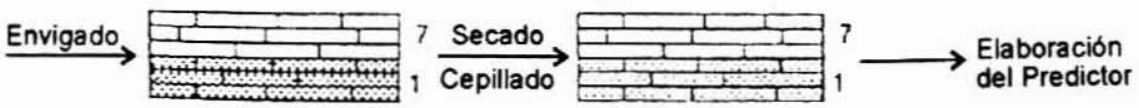

Figura $N^{\circ} 1$. METODOLOGÍA EMPLEADA EN LA CONFECCIÓN DEL PREDICTOR FERPA.

Con esa perspectiva. se relacionaron los volumenes obtenidos bajo las tres normas y se correlacionaron entre sí. Se obtuvo además regresiones y correlaciones para los volumenes clasificados para las tres normas y las distintas calidades A, B y C. Finalmente se ajustaron los modelos que explicaran con mayor confiabilidad los volumenes obtenidos. según las variables predictoras. 
El modelaje tridimensional, se obtuvo con la misma metodología ya expuesta, salvo que los defectos se llevaron a coordenadas superficiales con ejes X e Y. Con esta información se alimentó un sistema CAD que permitió obtener los modelos (Figura No2).
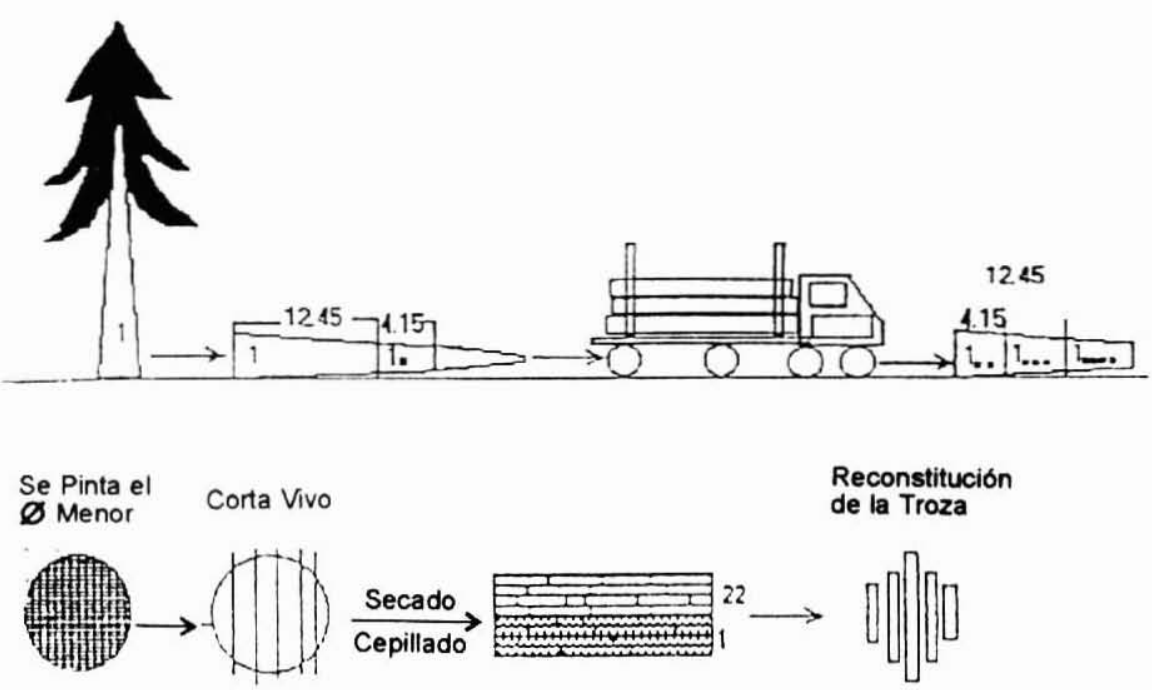

Tipo de Defecto

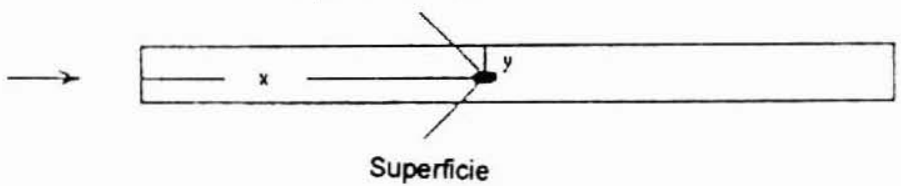

Figura No2. METODOLOGİA EMPLEAdA EN LA CONFECCIÓN DEL MAPEO TRIDIMENSIONAL.

\section{PRESENTACIÓN DE RESULTADOS}

A continuación se presentan los resultados de las distintas relaciones establecidas entre las variables. 
Cuadro $\mathrm{N}^{\circ} 4$.

VARIACIÓN PORCENTUAL DE CALIDADES A, B Y C EN FUNCIÓN DE LA CLASE DIAMÉTRICA.

\begin{tabular}{|c|c|c|c|c|c|c|}
\hline $\begin{array}{c}\text { Clase } \\
(\mathrm{cm})\end{array}$ & \multicolumn{4}{|c|}{ Dominante $\left({ }^{\circ} \mathrm{o}\right)$} & \multicolumn{3}{c|}{ Codominante (\%) } \\
\hline & A & B & C & A & B & C \\
\hline 16 & 9 & 79 & 12 & 7 & 93 & - \\
\hline 21 & - & 94 & 6 & 3 & 85 & 12 \\
\hline 26 & 8 & 82 & 10 & 8 & 81 & 11 \\
\hline 31 & 12 & 87 & 1 & 67 & 33 & - \\
\hline 36 & 18 & 81 & 1 & 29 & 64 & 7 \\
\hline 41 & 44 & 56 & - & - & - & - \\
\hline 46 & 19 & 79 & 2 & - & - & - \\
\hline
\end{tabular}

Cuadro $\mathrm{N}^{\circ} 5$.

VARIACIÓN PORCENTUAL DE CALIDADES ESTRICTURALES POR CLASIFICACIÓN SECUENCIAL EN FUNCIÓN DEL DIÁMETRO, ZONA A.

\begin{tabular}{|c|c|c|c|c|c|c|c|c|}
\hline Clase $(\mathrm{cm})$ & \multicolumn{4}{|c|}{ Dominante $\left({ }^{\circ}\right)$} & \multicolumn{5}{c|}{ Codominante (\%) } \\
\hline & ESS & ES1 & ES2 & ES0 & ESS & ES 1 & ES2 & ES0 \\
\hline 16 & 67 & 23 & - & - & 50 & 50 & - & - \\
\hline 21 & 59 & 31 & 10 & - & - & - & - & - \\
\hline 26 & - & - & - & - & 62 & 33 & 5 & - \\
\hline 31 & 61 & 29 & 10 & - & 62 & 29 & 9 & - \\
\hline 36 & 78 & 22 & - & - & - & 57 & 43 & - \\
\hline 41 & - & - & - & - & - & - & - & - \\
\hline 46 & 37 & 30 & 33 & - & - & - & - & - \\
\hline
\end{tabular}

- ESS: mejor grado norma estructural

ES1: bueno

ES2: bajo

ESO: eliminado

Cuadro $\mathrm{N}^{\circ} 6$.

VARIACIÓN PORCENTIAL DE IAAS CILIDADES SHOP POR CLASIFICACIÓN SECUENCIAL EN FUNCIÓN DEL. DIAMETRO, ZONA A.

\begin{tabular}{|c|c|c|c|c|c|c|c|c|}
\hline Clase $(\mathrm{cm})$ & \multicolumn{4}{|c|}{ Dominante $(\%)$} & \multicolumn{4}{c|}{ Codominante (\%) } \\
\hline & $\mathrm{SHI} \cdot$ & $\mathrm{SH} 2$ & $\mathrm{SH} 3$ & $\mathrm{SHO}$ & $\mathrm{SHI}$ & $\mathrm{SH} 2$ & $\mathrm{SH} 3$ & SHO \\
\hline 16 & - & - & 33 & 67 & - & - & 50 & 50 \\
\hline 21 & - & 7 & 44 & 49 & - & - & - & - \\
\hline 26 & - & - & - & - & - & 22 & 60 & 18 \\
\hline 31 & 2 & 32 & 42 & 24 & - & 29 & 51 & 20 \\
\hline 36 & - & - & 68 & 32 & - & - & - & 100 \\
\hline 41 & - & - & - & - & - & - & - & - \\
\hline 46 & - & 15 & 85 & - & - & - & - & - \\
\hline
\end{tabular}

- SHl: mejor grado norma shop

$\mathrm{SH} 2$ : bueno

SH3: bajo

SHO: eliminado 
Cuadro $\mathrm{N}^{\circ} 7$.

VARIACIÓN PORCENTUAL DE CALIDADES ESTRUCTURALES POR CLASIFICACIÓN SECUENCIAL EN FUNCIÓN DEL DIÁMETRO, ZONA B.

\begin{tabular}{|c|c|c|c|c|c|c|c|c|}
\hline Clase $(\mathrm{cm})$ & \multicolumn{4}{|c|}{ Dominante (\%) } & \multicolumn{4}{c|}{ Codominante (\%) } \\
\hline & ESS & ES1 & ES2 & ES0 & ESS & ES1 & ES2 & ES0 \\
\hline 16 & - & 34 & 66 & - & 18 & 31 & 51 & - \\
\hline 21 & 18 & 42 & 39 & 1 & - & - & - & - \\
\hline 26 & 3 & 55 & 40 & 2 & 8 & 59 & 31 & 2 \\
\hline 31 & 15 & 47 & 35 & 3 & 8 & 63 & 29 & - \\
\hline 36 & 23 & 41 & 36 & - & 12 & 47 & 36 & 5 \\
\hline 41 & - & - & - & - & - & - & - & - \\
\hline 46 & 12 & 28 & 60 & - & - & - & - & - \\
\hline
\end{tabular}

Cuadro N'8.

VARIACIÓN PORCENTUAL DE LAS CALIDADES SHOP POR CLASIFICACIÓN SECUENCIAL EN FUNCIÓN DEL DIÁMETRO, ZONA B.

\begin{tabular}{|c|c|c|c|c|c|c|c|c|}
\hline Clase (cm) & \multicolumn{5}{|c|}{ Dominante (\%) } & \multicolumn{4}{c|}{ Codominante (\%) } \\
\hline & SH1 & SH2 & SH3 & SHO & SH1 & SH2 & SH3 & SHO \\
\hline 16 & - & - & - & 00 & - & - & 14 & 86 \\
\hline 21 & - & 4 & 19 & 77 & - & - & - & - \\
\hline 26 & - & 5 & 26 & 69 & - & 4 & 33 & 63 \\
\hline 31 & - & 11 & 33 & 56 & - & 4 & 24 & 72 \\
\hline 36 & - & 16 & 35 & 59 & 5 & 22 & 15 & 58 \\
\hline 41 & - & - & - & - & - & - & - & - \\
\hline 46 & 8 & 13 & 52 & 27 & - & - & - & - \\
\hline
\end{tabular}

Para establecer las regresiones adecuadas. se codificó el número de trozas con los volúmenes totales, por grupo y por cada norma. Como variables de entrada se consideraron clase diamétrica, grado de dominancia y la zona de clasificación A y B, ya que la $\mathrm{C}$ queda reducida. en gran parte a cuartones. Las mismas correlaciones sirvieron para estimar el predictor FERPA.

\section{Cuadro $\mathrm{N}^{\circ} 9$. \\ REGRESIONES DE ENTRADA}

\begin{tabular}{|l|c|c|c|}
\hline Tipo de dominancia & Variable independiente & Variable dependiente & $\mathrm{r}^{2}$ \\
\hline Dominante & Clase diamétrica & Zona A & 0.84 \\
\hline Dominante & Clase diamétrica & Zona B & 0.99 \\
\hline Codominante & Clase diamétrica & Zona A & 0.86 \\
\hline Codominante & Clase diamétrica & Zona B & 0.99 \\
\hline
\end{tabular}


Cuadro $\mathrm{N}^{\circ} 10$.

\section{REGRESIONES DE SALIDA}

\begin{tabular}{|c|c|c|c|}
\hline Tipo de dominancia & Variable independiente & Variable dependiente & $r^{2}$ \\
\hline Dominante & $\mathrm{A}$ & SHI & 0.82 \\
\hline Dominante & $\mathrm{A}$ & $\mathrm{SH} 2$ & 0.86 \\
\hline Dominante & $\mathrm{A}$ & SH3 & 0.94 \\
\hline Dominante & A & $\mathrm{SHO}$ & 0.77 \\
\hline Dominamte & A & ESS & 0.98 \\
\hline Dominante & $\mathrm{A}$ & ESI & 0.99 \\
\hline Dominante & A & ES2 & 0.68 \\
\hline Dominante & $A$ & $\overline{\mathrm{ES} 0}$ & 1.00 \\
\hline Dominante & B & SHI & $0.02^{*}$ \\
\hline Dominante & $\mathrm{B}$ & $\mathrm{SH} 2$ & 0.59 \\
\hline Dominante & $\mathrm{B}$ & $\mathrm{SH} 3$ & 0.81 \\
\hline Dominante & $\mathrm{B}$ & SH0 & 0.93 \\
\hline Dominante & $\mathrm{B}$ & ESS & 0.93 \\
\hline Dominante & $\mathrm{B}$ & $\overline{E S 1}$ & 0.98 \\
\hline Dominante & $\mathrm{B}$ & ES2 & 0.98 \\
\hline Dominante & $\mathrm{B}$ & ES0 & 0.88 \\
\hline Codominante & $A$ & SHI & 1.00 \\
\hline Codominante & $A$ & $\mathrm{SH} 2$ & 0.96 \\
\hline Codominante & $\mathrm{A}$ & SH3 & 0.76 \\
\hline Codominante & $A$ & SHO & 0.65 \\
\hline Codominante & $A$ & ESS & 0.99 \\
\hline Codominante & $\mathrm{A}$ & ES1 & 0.98 \\
\hline Codominante & A & ES2 & $0.32^{*}$ \\
\hline Codominante & $\mathrm{A}$ & ESO & 1.00 \\
\hline Codominante & $\mathrm{B}$ & SHI & $0.11^{*}$ \\
\hline Codominante & $\mathrm{B}$ & $\mathrm{SH} 2$ & 0.68 \\
\hline Codominante & B & $\mathrm{SH} 3$ & 0.99 \\
\hline Codominante & $\mathrm{B}$ & $\mathrm{SHO}$ & 0.99 \\
\hline Codominante & $B$ & ESS & 0.99 \\
\hline Codominante & $B$ & ES1 & 0.99 \\
\hline Codominante & $\mathrm{B}$ & ES2 & 0.99 \\
\hline Codominante & $\mathrm{B}$ & ESO & 0.75 \\
\hline
\end{tabular}

* Se asume un volumen igual a cero por la naturaleza de los grupos

\section{DISCUSIÓN DE RESULTADOS}

Los comportamientos de calidades son homogéneos en la zona $\mathrm{B}$, independiente de la clase diamétrica, participa con un $76 \%$ del volumen total La zona $C$ varía de 6 a $8 \%$, pero no se considera. ya que se destina preferentemente a cuartones. La zona A participa con $16 \%$ promedio, como volumen de las trozas.

En la medida que los diámetros aumentan. los volumenes desclasificados se incrementan, ya que la cantidad de nudos sueltos sigue la misma tendencia. Esta 
tendencia se confirma con lo expuesto por Lavery(1986), que diferencia la madera en zona externa, de nudos sueltos, una zona intermedia, de nudos vivos, y el corazón al interior.

La médula no produce desclasificación, ya que va incluida en los cuartones. En las primeras trozas los nudos son pequeños y firmes, lo cual deriva en calidades buenas a muy buenas, que corresponden a ESI y ESS, respectivamente.

En la tercera troza, 8 a $10 \mathrm{~m}$ de altura, las ramas son mayores y se observa una disminución de calidad en las piezas (ES2). Esta calidad tiende a mejorar en las trozas superiores, en las cuales las ramas disminuyen su tamaño y son vivas.

La calidad más homogénea, se encuentra en la zona $B$ (Cuadro $N^{\circ} 7$ ) de las trozas, debido a que hacia el interior de las mismas, el tamaño de nudo disminuye, pero son más numerosos. Esto acorta el intervalo y decrece la presencia de ES1 y ES2. En la zona A. el $60 \%$ del volumen corresponde a ESS (Cuadro $N^{\circ} 5$ ), disminuyendo a $47 \%$ en la zona B.

En la clasificación Shop, que consiste en encontrar zonas "clear", aptas para una buena elaboración, se obtiene mucha desclasificación, independientemente de la zona de la troza o de su altura.

Calidades Shop 1, se presentan en cantidades pequeñas y sólo en clases diamétricas altas. preferentemente en la zona A. Los tipos Shop 2 y 3 aumentan en volumen debido a su menor estrictez, siendo casi equivalentes entre zonas A y B.

El tipo más homogéneo en su distribución, es el tipo Shop 3 que alcanza cerca del $50 \%$ para la zona A y $30 \%$ para la zona B (Cuadros $\mathrm{N}^{\text {os }} 6$ y 8 ).

El sistema de mapeo tridimensional, a través del predictor FERPA y las variables de salida, confirma las bondades del sistema CAD para predecir, sobre la base de variables del bosque la cantidad de volumen que se obtendrá para cada calidad.

\section{CONCLUSIONES}

Las calidades A, B y C en pino radiata, para la VII Región, se encuentran en proporciones de 16,76 y $8 \%$ respectivamente.

El $60 \%$ de la zona A y el $47 \%$ de la zona B, corresponden a calidad ESS. En lo referente a la zona C. el $100 \%$ es de calidad estructural y no clasifica para Shop. El $50 \%$ de la zona A y el $30 \%$ de B son Shop 3 .

Los altos grados de correlación alcanzados con las variables de salida permite utilizar el predictor FERPA y el mapeo tridimensional sin restricciones, salvo un muestreo previo del bosque en cuestión. 
El mapeo tridimensional abre grandes perspectivas para un mayor conocimiento del pino radiata y posibilita el uso de caracterizaciones tecnológicas, a través de simulación, para un mayor beneficio económico del productor de madera.

\section{RECONOCIMIENTOS}

Los autores desean dejar constancia, de su reconocimiento a Forestal CELCO, en especial a la División Estudios, representada en 1993, por los señores Wenceslao Sánchez y Leonardo Frola. con cuyo apoyo este trabajo pudo concretarse.

\section{REFERENCIAS BIBLIOGRÁFICAS}

Lavery, Peter., 1986. Plantation Forestry with Pinus radiata. New Zealand. FRI.

Wagner, Mario., 1992. Clasificación de la Madera Aserrada Estructural de Pinus radiata. CORMA. Curso de Madera Aserrada Estructural. Concepción.

Western Wood Products Association., 1990. Las Calidades Factory y Shop Proporcionan Ventajas Economicas. Traduccion.

Instituto Nacional de Normalización. NCh-178. Of79. Madera Aserrada de Pino Insigne. Clasificacion por Aspecto

Instituto Nacional de Normalización. NCh-1207. Of 90. Madera Aserrada de Pino Insigne Clasificación por Resistencia

Frola, L, Bustamante, F. y Croxatto, P., 1993. Metodologia de Cbservación para la Definición de Madera Secuencial. Documento Interno Forelco.

Kollman, F., 1959. Tecnologia de la Madera y sus Aplicaciones. Tomo I. INFIE. España.

Zandherg, J., 1988. Finite Element Fracture Prediction for Wood with Knots and Cross Grain. Wood and Fiher Science. Vol. 20.

Retamal, Alejandro., 1975 Anteprovecto de Norma de Clasificación de Madera en Trozas. UTE.

González M., J. y Pérez E., 1992. Relación Materia Prima-Producto. Informe Técnico. CELCO.

Boyce, J., 1961. Forest Pathology: Third Edition. Henry J. Vaux. 550 pags.

Del Pozo, H., 1984. Durabilidad de Algunos Factores Establecidos en la Norma ASTM y la Norma Británica. Santiago

INFOR., 1989. Principio de Organización y Operacion del Aserradero. Manual nº.

INFOR., 1979. La clasificación de la Madera. Informe Técnico $\mathrm{N}^{\circ} 52$. 